\title{
Coherence Resonance in Chaotic Electronic Circuits
}

\author{
Oscar Calvo $^{1}$, Claudio R. Mirasso ${ }^{1}$ and Raúl Toral ${ }^{1,2}$ \\ ${ }^{1}$ Departament de Física, Universitat de les Illes Balears, E-07071 Palma de Mallorca, Spain \\ ${ }^{2}$ Instituto Mediterráneo de Estudios Avanzados (IMEDEA), CSIC-UIB, E-07071 Palma de Mallorca, Spain
}

\begin{abstract}
We experimentally demonstrate that a chaotic electronic circuit exhibits the main features of coherence resonance. This phenomenon shows up as a quasi-periodic response when the circuit is subjected to an external white-noise source of the appropriate level.
\end{abstract}

Random fluctuations (or noise) usually constitute a source of disorder and are considered, in general, as detrimental in, e. g., linear information theory, electrical engineering or neurobiology. However, it has been shown in the recent past that the quality of the response of a system can be improved if a right amount of noise is added. One of the most well known examples is that of stochastic resonance [1], by which a system driven by an external signal optimizes its response for an intermediate value of the noise level. This behaviour has been observed in the information transfer in crayfish mechanoreceptors [2], tunnel diodes [3], electronic circuits [4], amongst other systems. More surprisingly, a quasi-periodic signal can be made to appear just by applying a noise source, without the need of an external forcing, to a system that, otherwise, exhibits a non-periodic behaviour. Again, the periodicity of the signal becomes optimal for some intermediate values of the noise intensity. This effect is called coherence resonance [5] and has been observed in excitable [5,6], bistable and oscillatory [7] and optical systems [8]. A similar phenomenon appears in systems close to a limit cycle [9].

In this letter we experimentally demonstrate that these ideas can be extended to chaotic systems, for which we find the main features of coherence resonance. Although we choose as an example a Chua circuit [10], we believe that the same behaviour should be observed in other chaotic systems with similar characteristics. Our Chua circuit is shown in figure 1. A digital acquisition board from National Instruments NIDAQ, sampling at $10 \mathrm{KHz}$, plugged into the PCMC/A slot of a laptop was used to digitize the signal. A Labview program controlled the board in a continuous acquisition mode and an HP 33120 function generator was used to provide the noise signals from 0 to $5 \mathrm{~V}$ [rms].

Mathematically, a Chua circuit is described in terms of three non-linear first order differential equations. These equations predict that the system has, for some range of parameters, three unstable fixed points. In this case, the output voltages $V_{1}$ and $V_{2}$ oscillate chaotically in time around different dynamical attractors. In our experiments, we choose $C_{1}=10$ $\mathrm{nF}, C_{2}=100 \mathrm{nF}, R=1673 \Omega, a=-8 / 7, b=-5 / 7$, where $a$ and $b$ characterize the slope of the non-linear Chua resistance (see inset fig. 1) [10]. For this set of parameters, there is a single scroll attractor (as shown in the fig. 1 (b)), and its mirror image (not shown in the figure). In the absence of any external perturbation, $V_{1}$ and $V_{2}$ chaotically oscillate around one of the unstable fixed points with a mean oscilla- tion frequency of $\sim 2.3 \mathrm{KHz}$ and without any possibility to jump to its mirror image attractor. However, when a small amount of noise is added to the circuit, $V_{1}$ and $V_{2}$ start to switch between the two single scroll attractors. Three typical time traces are shown in the figure 2 for the voltage $V_{2}$ at low, intermediate and large noise levels. As can be seen in panel (b) for the optimum noise level, the regularity of the jumping process becomes evident. This is the first indication that noise is inducing a more regular behaviour in this chaotic system.

To better quantify the situation we calculate the variance $\sigma$ of the residence time in the attractors normalized to the mean value $\langle t\rangle$. In figure 3 (a) it can be clearly seen that this quantity has a minimum (optimal periodicity) at an intermediate noise level, in this case for a noise voltage of $\sim 1.5 \mathrm{~V}[\mathrm{rms}]$. Another indicator has been also calculated: the normalized autocorrelation function of the time series, whose minimum value $C_{\min }$ measures the strength of the (anti)correlation between the residence time in the two states. As can be seen in figure 3 (b) this value of $C_{\min }$ reaches a minimum around the noise level of $1.5 \mathrm{~V}$ [rms], in agreement with the previous indicator. These results reveal the existence of a constructive effect of the noise that is capable to yield a maximum regularity in the process of transition from one chaotic attractor to the other.

In conclusion, we have given the first evidence of coherence resonance in a chaotic electronic circuit. It has been demonstrated experimentally that a quasi-periodic response can be induced by just applying an appropriate amount of noise to a circuit which, otherwise, exhibits a non-regular behaviour. The quality of the periodicity is quantified by the variance of the residence time and the minimum of the time correlation function. These results show that noise, instead of being a source of disorder, can actually increase the regularity in the output of an electronic, chaotic, circuit.

Acknowledgements This work has been supported by DGES (Spain) project PB97-0141-C02-01 and MCyT (Spain) project BMF2000-1108.

[1] L. Gammaitoni, P. Hänggi, P. Jung and F. Marchesoni, "Stochastic Resonance", Rev. Mod. Phys. 70, (1), pp. 223-287 (1998). 
[2] J. K. Douglass, L. Wilkens, E. Pantazelou and F. Moss, "Noise Enhancement of Information Transfer in Crayfish Mechanoreceptors by Stochastic Resonance", Nature 365, pp. 337-340 (1993).

[3] R. Mantegna and B. Spagnolo, "Stochastic Resonance in a Tunnel Diode”, Phys. Rev. E 49, (3), pp. R1792-R1795 (1994).

[4] X. Godivier, J. Rojas-Varela and F. Chapeau-Blondeau, "Noiseassisted signal transmission via stochastic resonance in a diode nonlinearity", Electron. Lett. 33, (20), pp. 1667-1667 (1997).

[5] A.S. Pikovsky and J. Kurths, "Coherence Resonance in a NoiseDriven Excitable System”, Phys. Rev. Lett. 78, (5), pp. 775-778 (1997).

[6] D.E. Postnov, S. K. Han, T. Yim, and O.V. Sosnovtseva, "Experimental Observation of Coherence Resonance in Cascaded Excitable Systems", Phys. Rev. E 59, (4), 3791-3794 (1999).

[7] B. Lindner and L. Schimansky-Geier, "Coherence and stochastic resonance in a two-state system", Phys. Rev. E 61, (6), pp. 6103-6110 (2000).

[8] G. Giacomelli, M. Giudici, S. Balle and J.R. Tredicce, "Experimental Evidence of Coherence Resonance in an Optical System”, Phys. Rev. Lett. 84, (15), pp. 3298-3301 (2000); J. Buldú, J. García-Ojalvo, C. Mirasso, M. C. Torrent and J. M. Sancho, "Effect of External Noise Correlation in Optical Coherence Resonance", http://arXiv.org/abs/nlin/0104047.

[9] H. Gang, T. Ditzinger, C. Z. Ning, and H. Haken, "Stochastic resonance without external periodic force", Phys. Rev. Lett. 71, (6), pp. 807-810 (1993); W. J. Rappel and S. H. Strogatz, "Stochastic resonance in an autonomous system with a nonuniform limit cycle", Phys. Rev. E 50, (4), pp. 3249-3250, (1994).

[10] "Chua's Circuit: A Paradigm for Chaos", R.N. Madan, ed. World Scientific Publishing (1993).

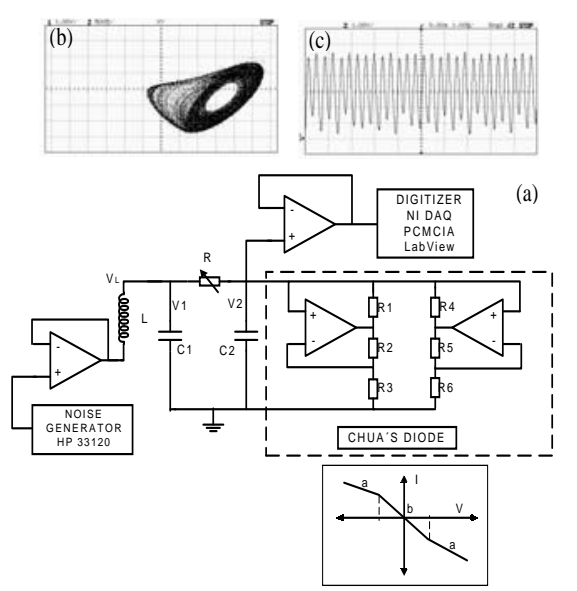

FIG. 1. (a) Block of the Chua Circuit. The inset plots the characteristics of the non-linear resistance; (b) Phase space representation $V_{1}$ vs. $V_{2}$; (c) Typical time trace of $V_{2}(t)$.

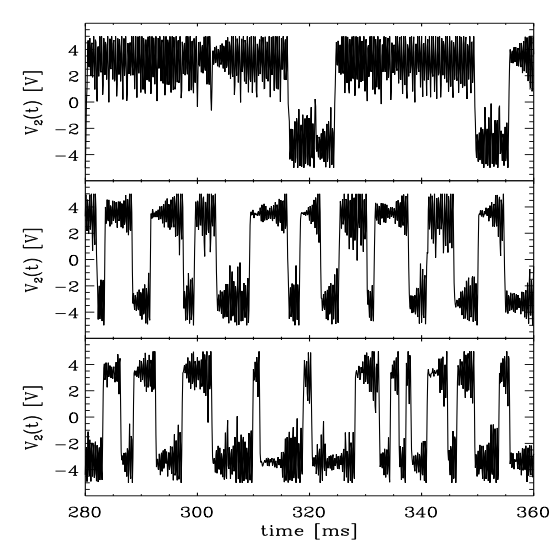

FIG. 2. Voltage time series for three different noise levels (maximum values): a) $0.5 \mathrm{~V}[\mathrm{rms}]$, b) $1.5 \mathrm{~V}[\mathrm{rms}]$, optimum noise level, and c) $2.5 \mathrm{~V}[\mathrm{rms}]$.

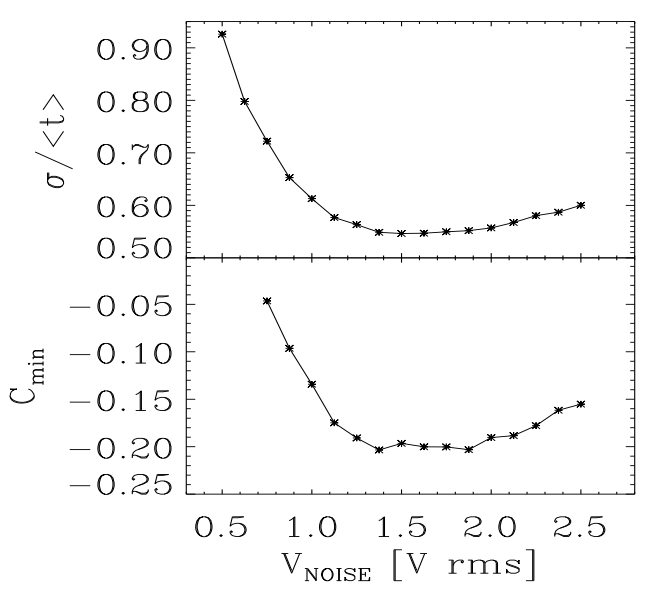

FIG. 3. (a) Variance of the residence time normalized by the mean time $\sigma /\langle T\rangle$ and (b) minimum of the correlation function $C_{\min }$ as a function of the noise level $V_{N O I S E}$. 